\title{
The Role of Electrostatic Charge in the Adhesion of Spherical Particles onto Planar Surfaces in Atmospheric Systems
}

\author{
Hyojin Kweon, ${ }^{\dagger}$ Sotira Yiacoumi, ${ }^{\dagger}$ and Costas Tsouris ${ }^{*}, \dagger, \S$ \\ ${ }^{\dagger}$ Georgia Institute of Technology, Atlanta, Georgia 30332-0373, United States \\ ${ }^{\S}$ Oak Ridge National Laboratory, Oak Ridge, Tennessee 37831-6181, United States \\ *Corresponding author: E-mail tsourisc@ornl.gov
}

Submitted for publication in

Colloids and Surfaces A: Physicochemical and Engineering Aspects

February 2015

Notice: This manuscript has been authored by UT-Battelle, LLC under Contract No. DE-AC0500OR22725 with the U.S. Department of Energy. The United States Government retains and the publisher, by accepting the article for publication, acknowledges that the United States Government retains a non-exclusive, paid-up, irrevocable, world-wide license to publish or reproduce the published form of this manuscript, or allow others to do so, for United States Government purposes. The Department of Energy will provide public access to these results of federally sponsored research in accordance with the DOE Public Access Plan (http://energy.gov/downloads/doe-public-access-plan). 


\title{
The Role of Electrostatic Charge in the Adhesion of Spherical Particles onto Planar Surfaces in Atmospheric Systems
}

\author{
Hyojin Kweon, ${ }^{\dagger}$ Sotira Yiacoumi, ${ }^{\dagger}$ and Costas Tsouris ${ }^{* \dagger, \$}$ \\ Georgia Institute of Technology, Atlanta, Georgia 30332-0373, United States \\ ${ }^{\S}$ Oak Ridge National Laboratory, Oak Ridge, Tennessee 37831-6181, United States
}

\begin{abstract}
The influence of electrostatic charge on the adhesive force between spherical particles and planar surfaces in atmospheric systems was studied using atomic force microscopy. Electrical bias was applied to modify the surface charge, and it was found that application of a stronger positive bias to a particle induces a stronger total adhesive force. The sensitivity of the system to changes in the bias depended on the surface charge density. For larger-size particles, the contribution of the electrostatic force decreased, and the capillary force became the major contributor to the total adhesive force. The influence of water adsorption on the total adhesive force and, specifically, on the contribution of the electrostatic force depended on the hydrophobicity of interacting surfaces. For a hydrophilic surface, water adsorption either attenuated the surface charge or screened the effect of surface potential. An excessive amount of adsorbed water provided a path to surface charge leakage, which might cancel out the electrostatic force, leading to a reduction in the adhesive force. Theoretically calculated forces were comparable with measured adhesive forces except for mica which has a highly localized surface potential. The results of this study provide information on the behavior of charged colloidal particles in atmospheric systems.
\end{abstract}

Keywords: Adhesive force, Electrostatic charge, Relative humidity, Particle adhesion 


\section{Introduction}

Interfacial forces such as van der Waals, capillary, and electrostatic forces may play an important role in atmospheric transport of aerosol particles, especially when the particle size is in the micrometer range. Among these forces, the electrostatic force is a major contributor to the behavior of charged particles. Radioactive particles are a relevant example because they acquire charge through direct self-charging, as well as indirect charging processes [1-5]. Arising from surface charge, the electrostatic force leads to transport of radioactivity far from radioactive sources and accumulation of contamination on soil and plant surfaces [6]. Electrical household appliances are a significant source of ultrafine charged particles, which affect indoor air quality [7-11]. Electrostatic interactions may prevent the aggregation of charged aerosol particles and allow them to carry various air pollutants including microorganisms, dust, and sometimes radon progeny to long distances [6]. Since charged particles are stabilized with respect to aggregation, their surface charge can enhance the transport of air pollutants. The surface charge may also increase the concentration of pollutants on surfaces, such as computer displays, printers, and power cables $[6,12]$.

Relative humidity $(\mathrm{RH})$ is another variable that affects the behavior of particles in the atmosphere. As the $\mathrm{RH}$ is increased, more water molecules adsorb onto surfaces, and a water meniscus may form between the interacting surfaces. Adsorption of water molecules promotes the adhesion of particles due to the surface tension of water and the pressure difference across the meniscus $[13,14]$. Since water is a polar molecule, which can be attracted by a charged surface, the presence of surface charge considerably affects water adsorption onto surfaces. It has also been reported that water adsorption contributes to charge build-up $[15,16]$, as well as dissipation of the charge by increasing the conductance of the material $[16,17]$. Thus, it is essential to consider not only the effects of surface charge and RH upon the adhesive force of micronand submicron- size particles in atmospheric systems, but also the relationship between surface charge and RH. 
Atomic force microscopy (AFM) has been used to study the interaction forces between micron-size particles and various substrate surfaces $[18,19]$. AFM is a suitable tool to study particle interaction forces in atmospheric systems because it enables simulation of the natural environment by controlling such variables as RH and surface potential. AFM has been used in our previous work for the study of micronand submicron-size particles including a silicon nitride cantilever tip, spherical silica particles of varying diameter, and bacterial spores with various planar surfaces [20-22]. The contribution of the electrostatic force was identified by scanning surface potential microscopy (SSPM) measurements and through comparisons of AFM force measurements with calculated values using mathematical models for the van der Waals, capillary, and electrostatic forces [23,24]. Kweon et al. measured the interacting forces of a silicon nitride tip with a radioactive gold $\left({ }^{198} \mathrm{Au}\right)$ surface [22]. It was found that the electrostatic force arising from the surface potential of ${ }^{198} \mathrm{Au}$ contributes to the total adhesive force. In addition to radioactive particles, there are many examples in which the electrostatic force plays a critical role because aerosol particles can naturally or artificially acquire surface charge $[6,7,12]$.

This study aims at investigating the contribution of the electrostatic charge and the influence of RH on the adhesive force of various materials. AFM was used to measure the adhesive force of silica and gold particles with various planar surfaces including mica, silica, and gold, which can be readily found in both natural and engineered environments. Mica and silica are insulating materials whereas gold is conductive, and mica can acquire a relatively higher surface charge density than silica does. In order to investigate the effect of particle size on the interaction forces, two different-size particles, $1 \mu \mathrm{m}$ and $2.5 \mu \mathrm{m}$ in diameter, were used. The interaction forces were also calculated using established models for the van der Waals, capillary, and electrostatic forces. Comparisons between experimental data and theoretical calculations in this work can be used to improve existing models. 


\section{Experimental and Modeling Methods}

\subsection{Surface preparation}

Mica, silica (fused quartz), and gold foil were used as planar surfaces in this study. Prior to cleaning, mica and silica were mounted onto a magnetic disc that adjusts substrate surfaces onto the AFM stage using two-sided adhesive tape. In order to minimize surface contamination that could result in the modification of surface properties, mica was cleaved just before each force measurement. Silica was stored in concentrated sulfuric acid for more than 24 hours and rinsed thoroughly with a large amount of deionized water. A pure gold foil of square shape was attached to the magnetic disc using silver paint, which enabled the application of electrical bias to the gold foil. The prepared gold kit was cleansed with ethanol and acetone, and rinsed thoroughly with a large amount of deionized water. Cleaned silica and gold surfaces were dried and stored in silica gel dehydrators for 3 to 5 days.

\subsection{AFM Probes}

AFM probes of a spherical silica particle and a gold particle were prepared for interaction force measurements. A single spherical silica particle was attached at the end of a v-shaped tipless cantilever made of silicon nitride. A gold probe was prepared by coating a silica probe with gold. Particle attachment and gold coating were performed by Novascan Technologies (Ames, Iowa). The procedure involved handling of the particles with micro-manipulators and attachment of a single particle on each cantilever with a thin film of glue. A gold coating film enabled the application of an electrical bias to the particle. Two different particle sizes $(1 \mu \mathrm{m}$ and $2.5 \mu \mathrm{m})$ were used to prepare both probes of silica and gold particles.

\subsection{AFM Force Measurements}

The interaction force between three different substrate surfaces (mica, silica, and gold) and the prepared particle probes was measured by using the AFM MultiMode V (Veeco Instruments, Plainview, New York) 
at room temperature $\left(19-21^{\circ} \mathrm{C}\right)$. Application of the electrical bias is allowed through the substrate surface and the particle probe. Since mica and silica are both electrically insulating materials, the electrical bias was introduced through the gold particle. For the system consisting of gold foil, electrical bias was applied through either the gold foil or the gold-coated particle. A silica particle probe was also used in the measurements with the gold foil. The magnitude of the electrical bias was controlled by changing one of the AFM operating parameters. The deflection of the particle probe due to the interaction forces between the probe and the surface was recorded in volts $(\mathrm{V})$ and converted to force $(\mathrm{nN})$ using Hooke's law: $F=k$ $\times S \times \Delta V$, where $F, k, S$, and $\Delta V$ are the force, spring constant, photodiode sensitivity, and change in deflection signal, respectively. The relative humidity (RH) was controlled in a cylindrical glass chamber that completely covered the AFM head and scanner.

The normal load $\left(F_{n}\right)$, adhesive force $\left(F_{a d}\right)$, and total adhesive force $\left(F_{t o t}\right.$, i.e., the sum of normal load and adhesive force) can be estimated from the force-distance curve obtained by AFM. Figure 1 shows a typical force-distance curve for two cases: interaction between a particle and a surface without a longrange attraction (solid line) and with a long-range attraction (broken line). In general, the normal load $\left(F_{n}\right)$ is the difference between the set-point, one of the AFM operating parameters, and point $\mathrm{d}$. The adhesive force $\left(F_{a d}\right)$ is the difference between points $\mathrm{c}$ and d. With the existence of the long-range attraction, however, it is not easy to distinguish $F_{n}$ from the curve due to the influence of long-range attraction on both $F_{n}$ and $F_{a d}$. Moreover, the probe can be deflected until the force measurement is completed, depending on the strength of the long-range attraction. In this work, therefore, $F_{n}$ is defined as the difference between the set-point and point d (or d' if a long-range attraction exists). The comparison of $F_{n}$ for different experimental conditions shows the influence of electrical bias applied to either the particle or the substrate surface. It is also more appropriate to compare $F_{t o t}$, the difference between the set-point and point c, instead of $F_{a d}$ because it is difficult to distinguish how much the electrical bias or RH influences $F_{n}$ and $F_{a d}$, respectively. 


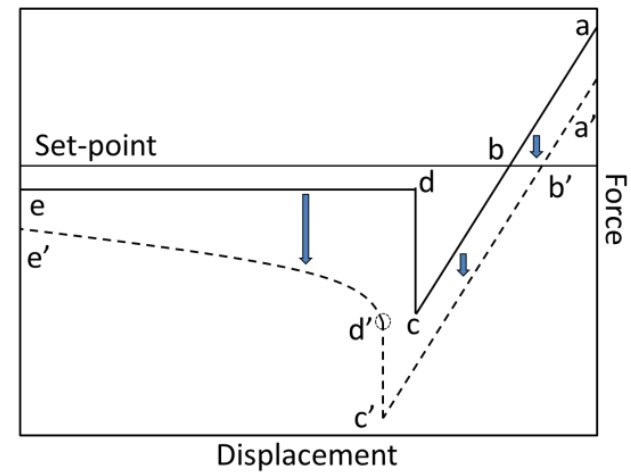

Figure 1. A typical retracting force-displacement curve (solid) and the curve with the presence of a longrange attraction (broken). The particle probe travels from a to e. The probe maintains contact with the surface due to the adhesive force (b to c). When the adhesive force is overcome, the particle is detached from the surface ( $\mathrm{c}$ to $\mathrm{d}$ ). As the probe moves further, the deflection of the probe returns to its original state ( $\mathrm{d}$ to e) and is maintained until the probe stops traveling. When a long-range attraction exists, the curve shifts towards the attraction region, and the deflection of the probe gradually returns to its original state (d' to e').

\subsection{Force Calculations}

To investigate the role of surface potential in the adhesion of a spherical particle on a planar surface, as shown in Figure 2, the electrostatic force was theoretically calculated and compared to measured forces. For simplicity, a smooth surface was assumed for both the particle and the substrate surface. The electrostatic force is calculated by Coulomb's law. For a system containing a substrate surface of large dimensions compared to a particle, the electric filed $(E)$ is given by $E=\sigma / 2 \varepsilon_{o}$, where $\sigma$ and $\varepsilon_{o}$ are the surface charge density and vacuum permittivity, respectively. The electrostatic force $\left(F_{e l}\right)$ applied to a particle with electrostatic charge $(q)$ in an electric field is given by $F_{e l}=E \cdot q=\left(\sigma / 2 \varepsilon_{o}\right) q$. Since the surface charge is the product of surface charge density $\left(\sigma_{i}\right)$ and surface area of the particle $\left(A_{\text {particle }}\right)$, the final equation of the electrostatic force is given by: 


$$
F_{e l}=-\frac{\sigma_{\text {substrate surface }} \sigma_{\text {particle }}}{2 \varepsilon_{0}} A_{\text {particle }}
$$
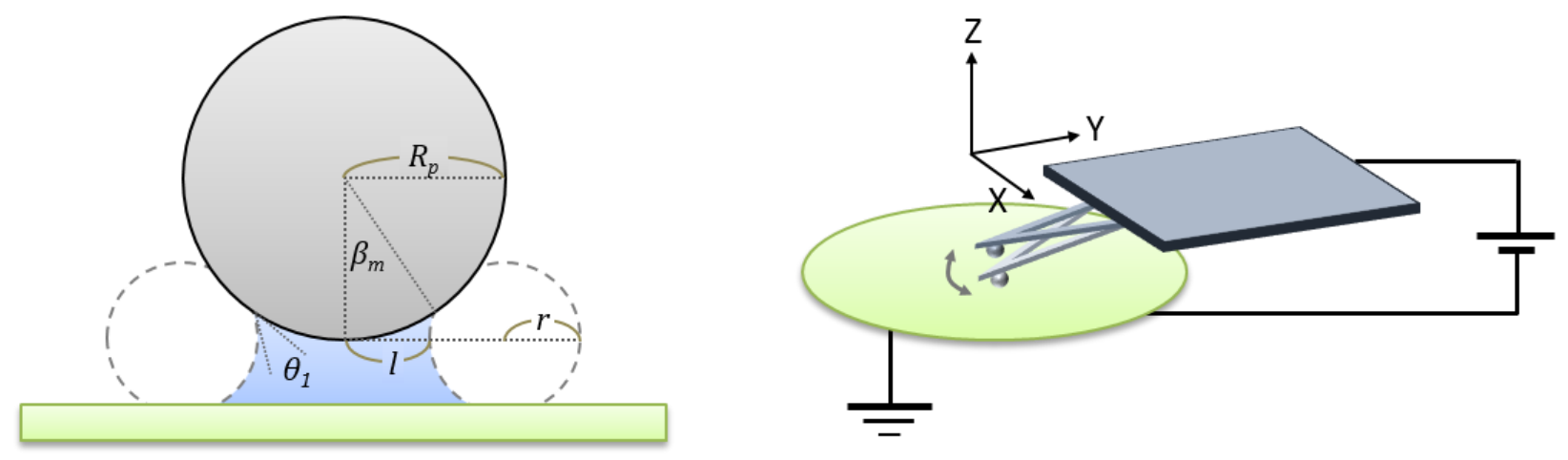

Figure 2. Simple schematic for the adhesion of a spherical particle on a planar surface (left) and the planar surface/particle system (right) for the force measurements using AFM.

If a dielectric medium exists between two interacting surfaces, the relative permittivity $(\varepsilon)$ is used instead of the vacuum permittivity. The relative permittivity is the product of the dielectric constant and the vacuum permittivity. For the calculation of $F_{e l}$, the values of surface potential for mica and silica at different RH levels were taken from the literature and our own measurements [17,23,24], and were either interpolated or extrapolated (Table 1).

Table 1. Charge density of mica and silica with respect to RH.

\begin{tabular}{|ccc|}
\hline RH $(\%)$ & \multicolumn{2}{c|}{ Charge density $\left(\mu \mathrm{C} / \mathrm{cm}^{2}\right)$} \\
& Mica & Silica \\
\hline 0 & -0.0030 & -0.01 \\
40 & -0.0019 & -0.05 \\
80 & -0.0017 & -0.09 \\
\hline
\end{tabular}


The van der Waals force $\left(F_{v d W}\right)$ was calculated using the following equation [25]:

$$
F_{v d w}=\frac{H_{\text {water }} R_{p}}{6 a^{2}}+\frac{H_{\text {air }}-H_{\text {water }}}{6 a^{2}} R_{p} \times \frac{a^{2}}{\left(a+R_{p}-R_{p} \cos \beta_{m}\right)^{2}}
$$

Here $H_{\text {water }}$ and $H_{\text {air }}, a, R_{p}$, and $\beta_{m}$ represent the Hamaker constant in water and air, intermolecular spacing, radius of the tip, and angle of meniscus, respectively.

The capillary $\left(F_{c a p}\right)$ force was obtained from the following relationships $[13,14]$ :

$$
\begin{aligned}
& F_{\text {cap }}=\pi l \gamma_{w}\left[-\frac{l R T}{\gamma_{w} V_{m}} \ln \left(\frac{P}{P_{o}}\right)+2 \sin \left(\theta_{1}+\beta_{m}\right)\right] \\
& \text { where } l=R_{p} \sin \beta+r\left[\sin \left(\theta_{1}+\beta_{m}\right)-1\right] \text { and } r=\frac{R_{p}\left(1-\cos \beta_{m}\right)+a}{\cos \left(\theta_{1}+\beta_{m}\right)+\cos \theta_{2}}
\end{aligned}
$$

where $\gamma_{w}, R, T, V_{m}, \theta_{1}, \theta_{2}$, and $P / P_{o}$ denote surface tension of water, ideal gas constant, temperature, molar volume of water, contact angle of the particle surface, contact angle of the substrate surface, and RH, respectively.

\section{Results}

AFM force measurements revealed the influence of both the electrostatic charge (through the electrical bias application to the system) and the RH on the interaction of a spherical particle, such as gold or silica, with various substrate surfaces, such as mica, silica, and gold. The electrostatic force was theoretically calculated as described in the Experimental and Modeling Methods section, using eq 1. To investigate the contribution of the electrostatic force to the total adhesive force, the calculated electrostatic force was added to the experimentally measured force at $0 \mathrm{~V}$ at each level of RH. For the force calculations, the surface charge densities of mica and silica were obtained from literature reports [17,23,24], and those of the gold particle or gold surface were calculated using the Gauss's Law [25]. 


\subsection{Normal Load}

When an electrical bias is applied to the system, a long-range attraction is observed in both approaching and retracting force-displacement curves. In general, these curves overlap each other, except for the part that represents the adhesion in the retracting curve (b'-d' in Figure 1), unless other events occur that can change the surface properties at the contact between the particle and the surface. As mentioned in the Experimental and Modeling Methods section, the normal load $\left(F_{n}\right)$ is estimated from the retracting curve. As the long-range attraction becomes stronger, the curve shifts towards the attractive region, and therefore the deflection is increased (c'-e' in Figure 1). Thus, $F_{n}$ is greater for the stronger long-range attraction. In addition, the poor overlap of approaching and retracting curves implies that the contribution of the electrical potential of interacting surfaces to the total adhesive force is changed after contact is made between the particle and the substrate surface due to modification of the surface properties, probably due to charge attenuation and charge leakage. This case is described in the Total Adhesive Force section.

Figure 3 shows randomly chosen data of average normal load values for systems with mica, silica, and gold substrate surfaces, respectively. In most cases, the normal load increased with an increase of the applied positive bias to gold due to a stronger electrical attraction with the negatively charged surfaces of mica and silica. The change in the interaction of a $1-\mu \mathrm{m}$ diameter particle with increasing bias was stronger than that of a $2.5-\mu \mathrm{m}$ diameter particle as the $\mathrm{RH}$ was increased from 0 to $80 \%$. This difference can be attributed to the large surface-to-volume (S/V) ratio of the $1-\mu \mathrm{m}$ particle, which is 2.5 times greater than that of the $2.5-\mu \mathrm{m}$ particle. The calculated surface charge density was also higher for the smaller particle. Thus, the $1-\mu \mathrm{m}$ diameter particle responded more strongly to the change in the electrical bias. A similar observation was made by Elfström et al. who reported that smaller nanowires are more sensitive to local charge changes [26].

The influence of $\mathrm{RH}$ on the normal load $\left(F_{n}\right)$ depends on the hydrophobicity or the contact angle of the interacting surfaces. Adsorption of water molecules can increase the surface charge density depending on 
material properties. For example, when water molecules are adsorbed, silica becomes negatively charged due to the formation of silanol groups, and thereby the density of negative charge on the surface is increased with increasing RH $[15,17,24]$. Guoveia et al. also reported charge build-up on silica surface due to water adsorption [15]. When an excessive amount of water molecules is adsorbed at a high RH, however, the probability for charge dissipation increases. Our results show that $F_{n}$ decreases with an increase of RH regardless of the applied bias level for systems with mica, whereas a different phenomenon occurs in systems with silica or gold as substrate surfaces. Due to the highly hydrophilic nature of mica, the amount of adsorbed water molecules on mica is much higher than the amount adsorbed on silica or gold at the same level of RH, leading to the dissipation of surface charge and thereby the reduction of $F_{n}$.
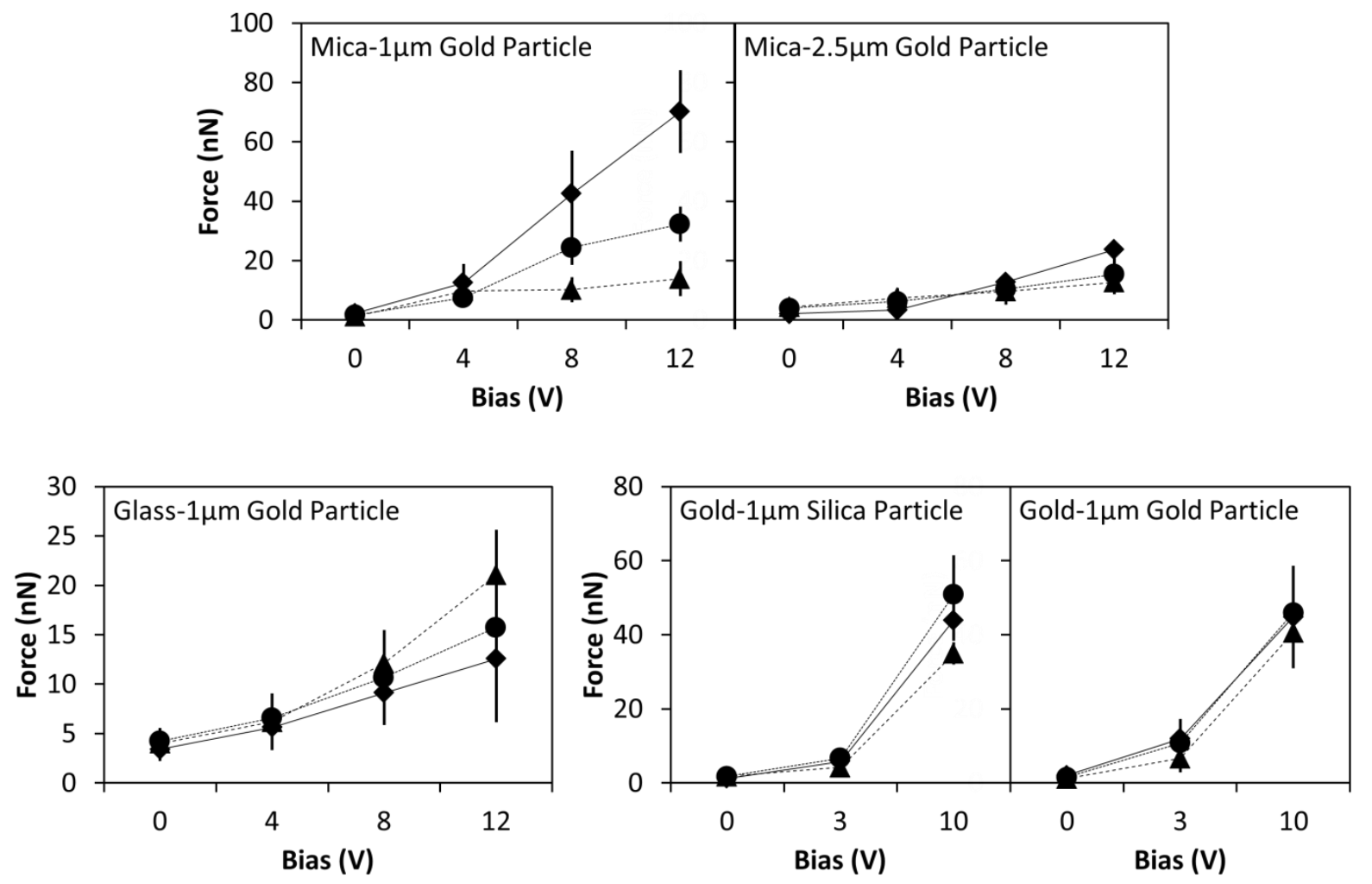

Figure 3. Average measurements of the normal load of the interaction of various planar surfaces with gold or silica particles. Diamonds, circles, and triangles represent measurements at 0,40 , and $80 \%$ of RH, respectively. 
Contrary to the general trend of the variation of the normal load $\left(F_{n}\right)$ with the electrical bias, experimental data for mica at $0 \% \mathrm{RH}$ showed a decrease in $F_{n}$ with increasing bias (Figure 4 ). Mica acquires potassium ions between its unit layers, which balance the net charge between the layers. When mica is cleaved, potassium ions move to either side of the layers and make the freshly cleaved mica either positively or negatively charged [22,27-29]. The surface charge of mica is highly localized due to the irregular removal of potassium ions, as well as the random cleavage rate [30]. Therefore, the observations at $0 \% \mathrm{RH}$ imply that the surface charge of mica is positive for the set of experiments shown in Figure 4, whereas mica is negatively charged for the experiments shown in Figure 3. When RH is increased to 40\%, however, the change of the normal force with the bias follows the general behavior. When the freshly cleaved mica is covered with water, potassium ions are dissociated into water and the sign of surface charge becomes negative [31], indicating that electrostatic attraction increases with positive bias. At $80 \% \mathrm{RH}$, the increase of the bias does not influence further $F_{n}$ because the amount of adsorbed water molecules is enough to dissipate the surface charge of mica and shield the influence of surface potential.

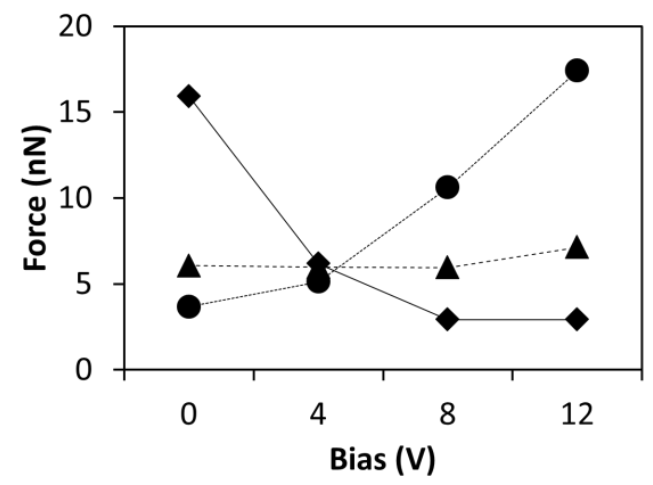

Figure 4. The normal load between mica and $2.5-\mu \mathrm{m}$ gold particles with an opposite trend to the one observed at $0 \%$ RH. Diamonds, circles, and triangles represent measurements at 0,40 , and $80 \% \mathrm{RH}$, respectively.

The electrostatic contribution of interacting surfaces and the influence of water adsorption are discussed further in the following section. 


\subsection{Total Adhesive Force}

The measured total adhesive force $\left(F_{t o t}\right)$ and the theoretically calculated values of the interaction force between mica and a 1- $\mu \mathrm{m}$ gold particle are compared in Figure 5. The total adhesive force increases with increasing electrical bias at 0 and $40 \% \mathrm{RH}$ due to the contribution of the electrostatic interaction. At $80 \%$ $\mathrm{RH}$, however, the force increases with an increase of the electrical bias from 0 to $4 \mathrm{~V}$ and then decreases with a further increase of the bias. Based on this result, it can be considered that the surface potential plays a role in the enhancement of water adsorption. The amount of increase in $F_{\text {tot }}$ was similar at 0 and $40 \% \mathrm{RH}$ when the bias increased from 0 to $4 \mathrm{~V}$, but the change in $F_{\text {tot }}$ due to the increase of the bias from 4 to $8 \mathrm{~V}$ was higher at $40 \% \mathrm{RH}$ than $0 \% \mathrm{RH}$. Since RH is the only difference between the two cases, it is considered that water molecules were attracted by surface charge induced by the bias application, due to the polarity of water molecules, resulting in an increase of the capillary contribution. Similarly, the increase in the $F_{\text {tot }}$ with an increase of the bias from 0 to $4 \mathrm{~V}$ was higher at $80 \% \mathrm{RH}$ than 0 and $40 \% \mathrm{RH}$, showing that the enhancement of water adsorption occurs at a weaker bias application for a higher RH level.

Beyond a certain concentration of adsorbed water molecules due to the increase of $\mathrm{RH}$, adsorbed water molecules screen the surface potential or attenuate the surface charge, resulting in the reduction of the electrostatic force $\left(F_{e l}\right)$ contribution. The increase of $F_{t o t}$ with increased bias from 8 to $12 \mathrm{~V}$ was lower at $40 \% \mathrm{RH}$ than $0 \% \mathrm{RH}$, indicating that adsorbed water molecules shielded the surface potential [32], resulting in the reduction of the $F_{e l}$ contribution. When the bias increased over $4 \mathrm{~V}$ at $80 \% \mathrm{RH}$, the $F_{\text {tot }}$ decreased. This behavior is believed to be due to charge leakage through the excessive amount of adsorbed water molecules. Charge leakage can be more clearly identified by comparing the forcedisplacement curve in the same set of experiments. As Figure 6 shows, the long-range attraction was significantly reduced after the first contact of the two surfaces during force measurements. This result suggests that the adsorbed water molecules formed a water layer upon the first contact between mica and 
gold particle. This layer remained on the particle surface, thereby it provided a channel for surface charge leakage during the second force measurement. As a result, at $80 \% \mathrm{RH} F_{\text {tot }}$ was reduced to the force value similar to the case when no electrical bias was applied.

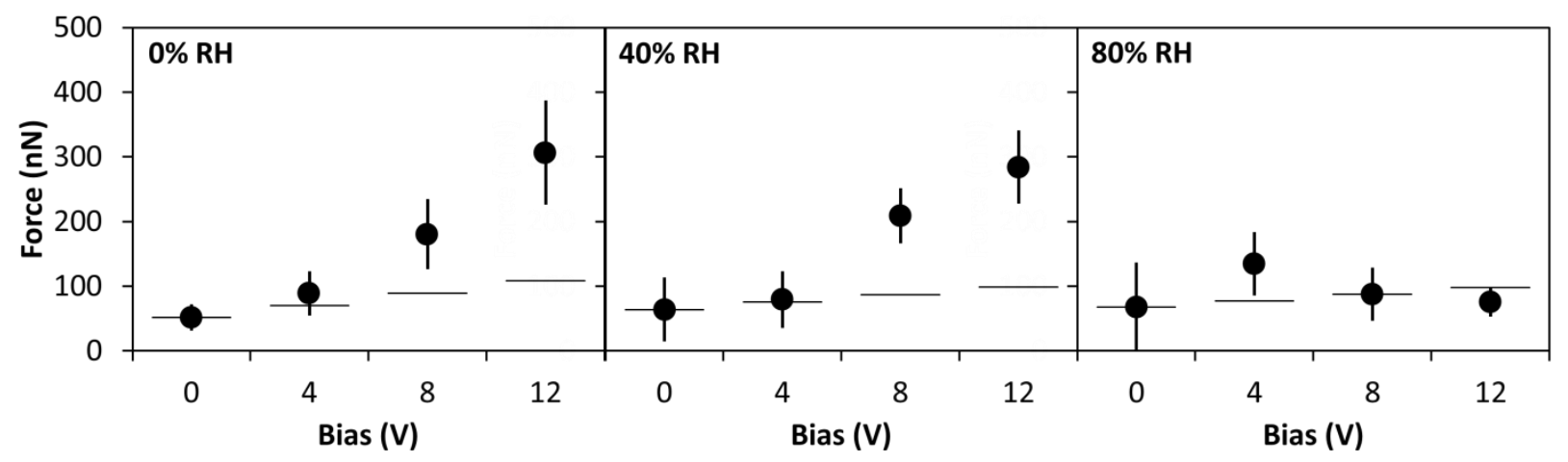

Figure 5. Average measurements of the total adhesive force (solid circles) and theoretical values (horizontal bars) of the interaction between mica and a $1-\mu \mathrm{m}$ diameter gold particle. The horizontal bars are the sums of the measured total adhesive force at $0 \mathrm{~V}$ and the theoretically calculated electrostatic force.

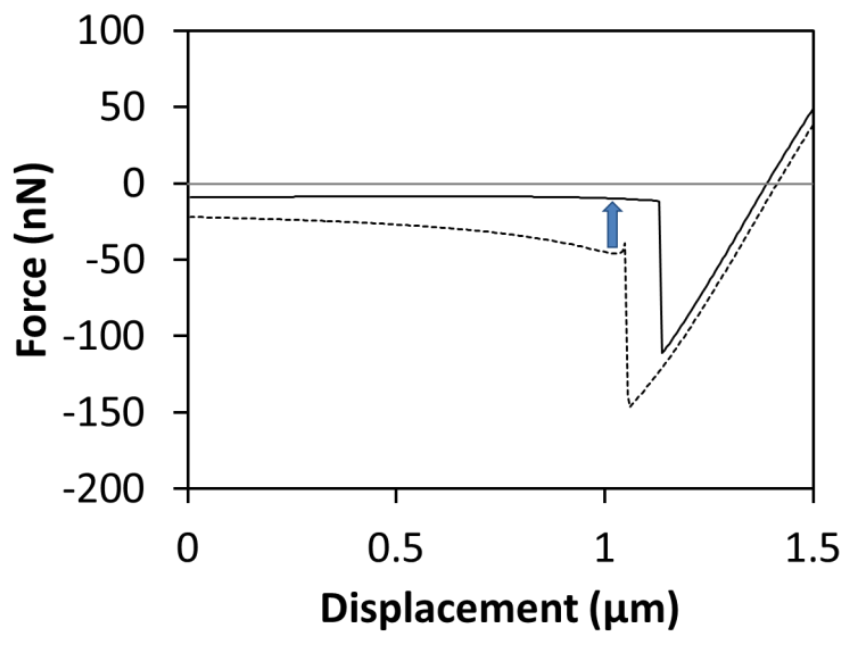

Figure 6. Force-displacement curves of the first (dotted line) and second (solid line) measurements in the same set of experiments. The decrease of the normal load and the total adhesive force in the second measurement indicate that charge leakage occurred at the contact of the gold particle and mica. 
The relative humidity also affects the total adhesive force $\left(F_{t o t}\right)$; in general, $F_{t o t}$ increases with increasing $\mathrm{RH}$, especially at 0 and $4 \mathrm{~V}$. Water adsorption contributed to $F_{\text {tot }}$ by increasing the capillary force. However, the contribution of RH was much smaller than that of the electrostatic force.

The contribution of the electrostatic force $\left(F_{e l}\right)$ was calculated by the theoretical model. To compare the theoretical forces with the experimental measurements, the calculated $F_{e l}$ was added to the measured $F_{t o t}$ at $0 \mathrm{~V}$ for each level of RH. The horizontal bars in Figure 5 are the sums of the measured forces at $0 \mathrm{~V}$ and the theoretical $F_{e l}$. The theoretical model can predict the increase of the force with the bias, but it underestimates the contribution of $F_{e l}$, except when charge leakage occurred. Since the surface charge density or surface potential of mica is highly localized, both positive and negative charges exist on the same surface of a freshly cleaved mica [30]. Moreover, the charge density of mica can vary according to the cleavage rate [30]. Thus, a poor reproducibility for the charge density of mica is expected. As a result, the theoretical model is only able to capture the general trend of the variation of force with the electrical bias. The model cannot describe the charge leakage occurring at 8 and $12 \mathrm{~V}$ at $80 \% \mathrm{RH}$ because it does not include terms describing the influence of water adsorption.

Figure 7 presents the total adhesive force $\left(F_{t o t}\right)$ between mica and a $2.5-\mu \mathrm{m}$ gold particle. It is shown that the force is not influenced by the bias application as much as the total adhesive force, found for the mica/1- $\mu \mathrm{m}$ gold particle system. The smaller contribution of $F_{e l}$ to the interaction force between mica and a $2.5-\mu \mathrm{m}$ gold particle was expected based on the results of normal load $\left(F_{n}\right)$ shown in Figure 3, where the magnitude of $F_{n}$ for the $2.5-\mu \mathrm{m}$ gold particle was much smaller than that for the $1-\mu \mathrm{m}$ gold particle. In addition, $F_{\text {tot }}$ was slightly decreased as the bias was increased from 0 to $4 \mathrm{~V}$. It is unclear what caused this behavior, but a repulsive effect is not likely because the presented results do not include data showing repulsion in the $F_{n}$. The total adhesive force was increased with a further increase of the bias voltage. Charge leakage was also observed at $12 \mathrm{~V}$ for $80 \% \mathrm{RH}$. The influence of $\mathrm{RH}$ on $F_{\text {tot }}$ was stronger than that of the bias on the interaction force between mica and a $2.5-\mu \mathrm{m}$ particle, and the magnitude of the 
force was larger than that of the interaction force between mica and a $1-\mu \mathrm{m}$ gold particle. This is opposite to the behavior of the $F_{n}$, which is independent from the capillary and van der Waals forces, implying that the capillary force is the major contributor to $F_{t o t}$ for the interaction of larger particles. The theoretical model describes the trend in $F_{\text {tot }}$, after $4 \mathrm{~V}$ bias was applied at 0 and $40 \% \mathrm{RH}$, but overestimates the magnitude of the force. As explained for the mica/1- $\mu \mathrm{m}$ gold particle system, the discrepancy is due to the poor reproducibility of the surface charge density of mica and the absence of terms in the model that account for the influence of water adsorption on the interacting surfaces.

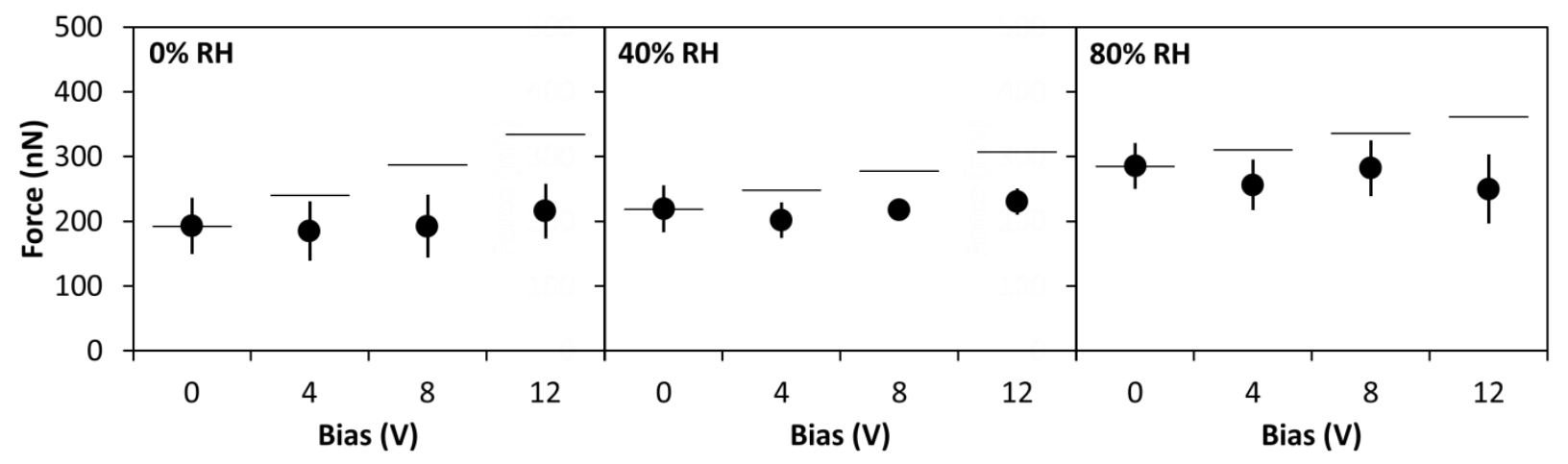

Figure 7. Average measurements of the total adhesive force (solid circles) and theoretical values (horizontal bars) of the interaction between mica and a $2.5-\mu \mathrm{m}$ diameter gold particle. The horizontal bars are the sums of the measured total adhesive force at $0 \mathrm{~V}$ and the theoretically calculated electrostatic force.

Figure 8 shows the results of the interaction of the fused silica with a $1-\mu \mathrm{m}$ gold particle. The total adhesive force $\left(F_{t o t}\right)$ between silica and a $1-\mu \mathrm{m}$ gold particle showed larger variations than that for mica/gold particles. This may be attributed to the heterogeneous surface properties of silica. It is reported that the surface potential of dielectric surfaces is heterogeneous $[16,17,33]$. Surface roughness may be another contributor to the variation because the silica surface also has a surface roughness of about $2 \mathrm{~nm}$ in RMS (root mean square), whereas mica is atomically flat [21]. 


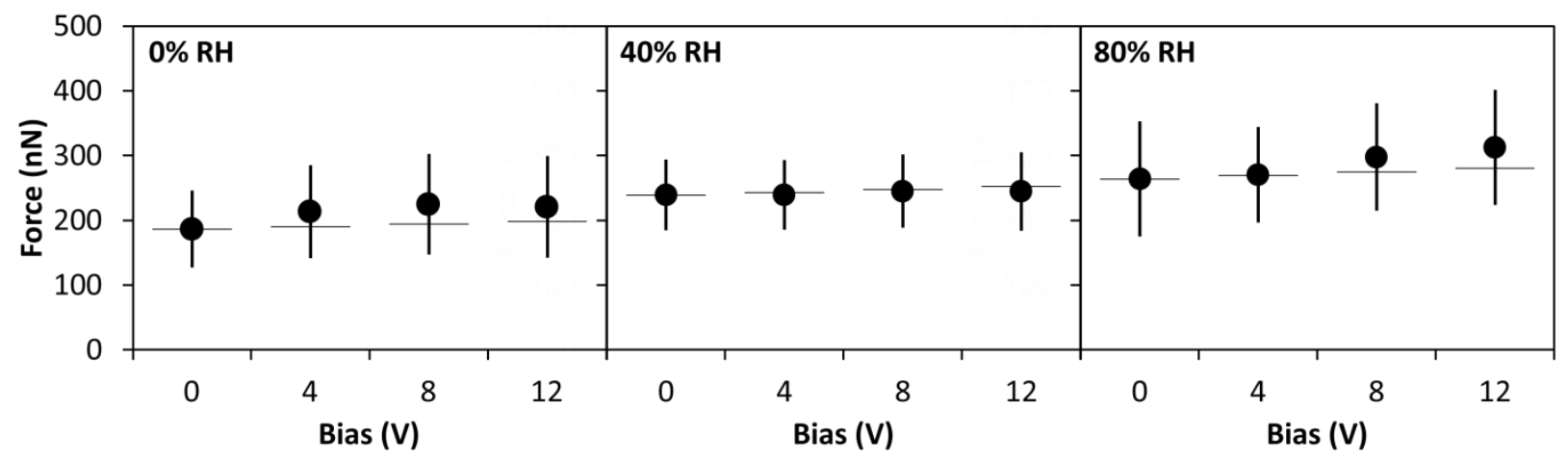

Figure 8. Average measurements of the total adhesive force (solid circles) and theoretical values (horizontal bars) of the interaction between silica and a 1- $\mu \mathrm{m}$ diameter gold particle. The horizontal bars are the sums of the measured total adhesive force at $0 \mathrm{~V}$ and the theoretically calculated electrostatic force.

As shown in Figure 8, the interaction force between silica and a 1- $\mu$ m gold particle was influenced by the increase of the bias application, but not as much as that between mica and a 1- $\mu \mathrm{m}$ gold particle. The increase of $F_{\text {tot }}$ from 0 to $12 \mathrm{~V}$ of the particle bias was up to $18 \%$ at 0 and $40 \% \mathrm{RH}$, but only $2 \%$ at $80 \%$ RH. This is due to the much lower surface charge density of silica than that of mica $[23,24]$. The total adhesive force was gradually increased with increasing bias at all RH levels, indicating that the amount of adsorbed water molecules on silica, even at $80 \% \mathrm{RH}$, was not enough to cause charge leakage. This behavior is different from the results on mica shown in Figure 5. Since silica is less hydrophilic than mica, it is thought that silica is not completely covered by a water layer [21]. A good agreement between the measured force data and the theoretical values for this system implies less interference between water adsorption and surface potential due to the lower hydrophilicity of silica than mica.

The total adhesive force $\left(F_{t o t}\right)$ for the interaction between silica and a $2.5-\mu \mathrm{m}$ gold particle was larger than that between silica and a 1- $\mu \mathrm{m}$ gold particle, but the trend in the force was inconsistent with either the electrical bias or the RH (results not shown). It is unclear what led to this result, but the heterogeneity of 
silica including surface roughness and electrostatic charge can be a possible reason it is difficult to reproduce or predict the surface potential of insulating materials [16].

For the gold foil interaction with a $1-\mu \mathrm{m}$ silica particle, the trend in the total adhesive force $\left(F_{t o t}\right)$ with the application of the electrical bias was similar to the interaction between silica and a $1-\mu \mathrm{m}$ gold particle. The total adhesive force increased with increasing bias as described by the theoretical model; it also increased with increasing RH (Figure 9). The magnitude of the force, however, was much smaller than that for the interaction between silica and the gold particle. Metals, including gold, have much higher Hamaker constant values (up to an order of magnitude higher) due to their high polarizability [34], thus the theoretical model predicts a relatively high adhesive force for the gold/1- $\mu \mathrm{m}$ silica particle system, about $220 \mathrm{nN}$ at $0 \mathrm{~V}$ and $0 \% \mathrm{RH}$, i.e., at conditions with no contributions from the capillary and electrostatic forces. The high surface energy of the gold surface, however, attracts various gas-phase impurities, including organic substances, leading to surface contamination and thus a decrease in the van der Waals force [34-37]. The total adhesive force for the gold-gold particle system was even smaller than that of the gold-silica particle (Figure 10). Accordingly, surface contamination of both the gold foil and the gold particle was suspected. The total adhesive force increased with RH, regardless of the bias level. However, a good agreement between the measurements and calculated values for the gold $/ 1 \mu \mathrm{m}$-silica particle system suggests that the influence of water adsorption on the contribution of the electrostatic force was small.

The total adhesive force between a gold substrate and a gold particle also increased with the electrical bias, but decreased with increasing RH. Theoretical models (eqs. 2 and 3) show that this trend can be found in the interaction between highly hydrophobic surfaces $[13,14,34]$. Therefore, the results indicate that the gold surface and gold particle have a higher contact angle than silica surfaces, i.e., gold is more hydrophobic than silica. 


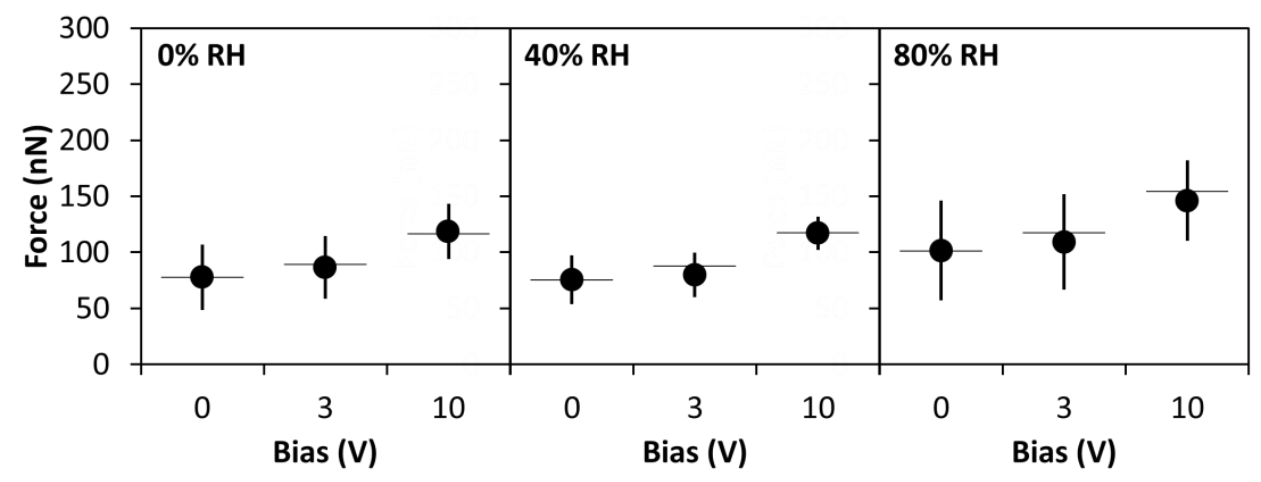

Figure 9. Average measurements of the total adhesive force (solid circles) and theoretical values (horizontal bars) of the interaction between gold and a $1-\mu \mathrm{m}$ diameter silica particle. The horizontal bars are the sums of the measured total adhesive force at $0 \mathrm{~V}$ and the theoretically calculated electrostatic force.

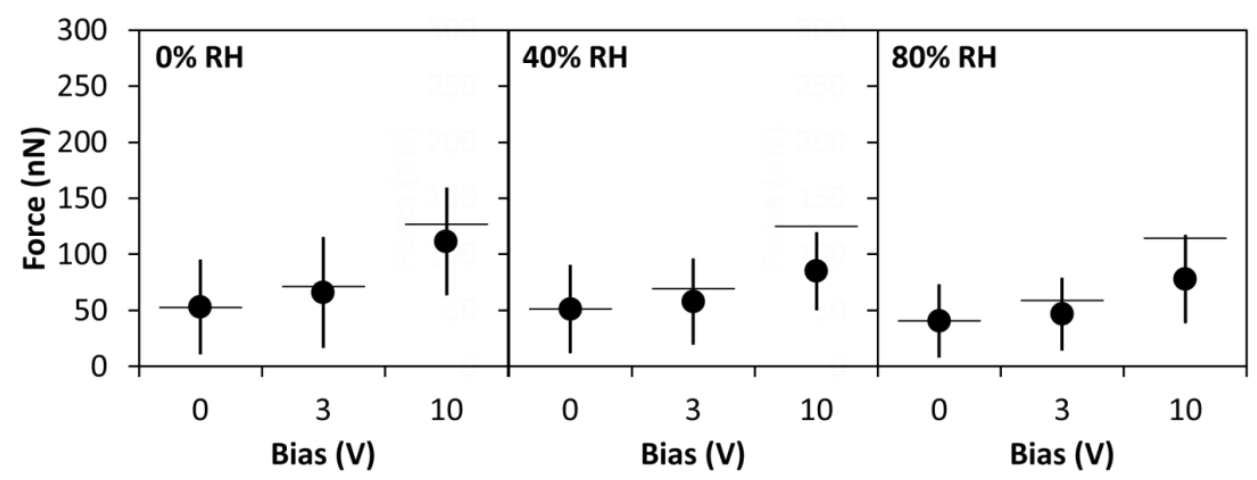

Figure 10. Average measurements of the total adhesive force (solid circles) and theoretical values (horizontal bars) of the interaction between gold and a $1-\mu \mathrm{m}$ diameter gold particle. The horizontal bars are the sums of the measured total adhesive force at $0 \mathrm{~V}$ and the theoretically calculated electrostatic force.

\section{Conclusions}

The influence of surface electrostatic properties on interaction forces in atmospheric systems was studied by measuring the total adhesive force of sphere-plane systems using AFM. Electrical bias was applied through either a gold particle for dielectric substrates or a gold foil to modify the surface potential and subsequently the electrostatic surface charge. For the mica/1- $\mu \mathrm{m}$ gold particle system, the contribution of 
the electrostatic force increases with the electrical bias, but the extent of the increase is diminished as RH is increased. At $80 \% \mathrm{RH}$, the total adhesive force decreases to the value of the adhesive force without bias applied due to charge leakage caused by an excessive amount of adsorbed water molecules. When the particle size is increased from $1 \mu \mathrm{m}$ to $2.5 \mu \mathrm{m}$, the influence of the bias decreases suggesting that the capillary force is the dominant contributor to the total adhesive force. When mica acquires positive charge upon cleavage, the influence of the bias is reversed due to the electrostatic repulsion between likely charged surfaces, but regains its general trend due to water adsorption. The total adhesive forces for the other systems with silica substrate-gold particle, gold substrate-silica particle, and gold substrate-gold particle also become stronger with increasing bias, but the change in the forces is smaller than that for the mica-gold particle system because silica and gold substrates acquire a smaller surface charge density. The influence of water adsorption on the contribution of the electrostatic force depends on the hydrophobicity or contact angle of the interacting surfaces.

Theoretical calculations of the interaction forces were obtained, and the electrostatic force was added to the measured force at $0 \mathrm{~V}$ for each level of $\mathrm{RH}$ for comparison with the total force at various levels of bias and RH. In most cases, the measured forces are comparable to the theoretically calculated values except for the mica-gold particle system because the surface potential of mica is localized and thus cannot be described by a single measurement [30]. The theoretical model does not include contributions from complex phenomena, such as charge attenuation or charge leakage, caused by water adsorption. The amount of water adsorption and its influence on the electrostatic properties of dielectric surfaces become important at high values of RH. Therefore, a charge leakage model needs to be developed to further improve the theoretical predictions. Results from this work may be useful for providing insight into the monitoring and control of micron- and submicron-size particles in atmospheric systems where the effects of surface electrostatic charge and RH become important. 


\section{Acknowledgment}

This work was supported by the Defense Threat Reduction Agency under grant number HDTRA1-08-10BRCWMD-BAA. The manuscript has been co-authored by UT-Battelle, LLC, under Contract No. DEAC05-00OR22725 with the U.S. Department of Energy.

\section{References}

[1] C.F. Clement, R.A. Clement, R.G. Harrison, Charge distributions and coagulation of radioactive aerosols, J. Aerosol Sci. 26 (1995) 1207-1225.

[2] C.F. Clement, R.G. Harrison, The charging of radioactive aerosols, J. Aerosol Sci. 23 (1992) 481-504.

[3] L.D. Reed, H. Jordan, J.A. Gieseke, Charging of radioactive aerosols, J. Aerosol Sci. 8 (1977) 457463.

[4] V.I. Yoschenko, et al., Resuspension and redistribution of radionuclides during grassland and forest

fires in the Chernobyl exclusion zone: part I. Fire experiments, J. Environ. Radioactiv. 86 (2006) 143-163.

[5] G. Lujaniene, V. Aninkevicius, V. Lujanas, Artificial radionuclides in the atmosphere over Lithuania, J. Environ. Radioactiv. 100 (2009) 108-119.

[6] H. Tammet, V. Kimmel, S. Israelsson, Effect of atmospheric electricity on dry deposition of airborne particles from atmosphere, Atmos. Environ. 35 (2001) 3413-3419.

[7] T. Salthammer, T. Schripp, E. Uhde, M. Wensing, Aerosols generated by hardcopy devices and other electrical appliances, Environ. Pollut. 169 (2012) 167-174.

[8] C. He, L. Morawska, L. Taplin, Particle emission characteristics of office printers, Environ. Sci. Technol. 41 (2007) 6039-6045.

[9] P. McGarry, L. Morawska, C.R. He, R. Jayaratne, M. Falk, Q. Tran, H. Wang, Exposure to particles from laser printers operating within office workplaces, Environ. Sci. Technol. 45 (2011) 6444-6452. 
[10] T. Schripp, I. Kirsch, T. Salthammer, Characterization of particle emission from household electrical appliances, Sci. Total Environ. 409 (2011) 2534-2540.

[11] L. Wallace, F. Wang, C. Howard-Reed, A. Persily, Contribution of gas and electric stoves to residential ultrafine particle concentrations between 2 and $64 \mathrm{~nm}$ : Size distributions and emission and coagulation rates, Environ. Sci. Technol. 42 (2008) 8641-8647.

[12] J.F. Kok, N.O. Renno, Enhancement of the emission of mineral dust aerosols by electric forces, Geophys. Res. Lett. 33 (2006).

[13] F.M. Orr, L.E. Scriven, A.P. Rivas, Pendular rings between solids: Meniscus properties and capillary force, J. Fluid Mech. 67 (1975) 723-742.

[14] X.D. Xiao, L.M. Qian, Investigation of humidity-dependent capillary force, Langmuir 16 (2000) 8153-8158.

[15] R.F. Gouveia, C.A.R. Costa, F. Galembeck, Electrostatic patterning of a silica surface: A new model for charge build-up on a dielectric solid, J. Phys. Chem. B 109 (2005) 4631-4637.

[16] R.F. Gouveia, C.A.R. Costa, F. Galembeck, Water vapor adsorption effect on silica surface electrostatic patterning, J. Phys. Chem. C 112 (2008) 17193-17199.

[17] R.F. Gouveia, F. Galembeck, Electrostatic charging of hydrophilic particles due to water adsorption, J. Am. Chem. Soc. 131 (2009) 11381-11386.

[18] H.J. Butt, R. Berger, E. Bonaccurso, Y. Chen, J. Wang, Impact of atomic force microscopy on interface and colloid science, Adv. Colloid Interfac. 133 (2007) 91-104.

[19] H.J. Butt, B. Cappella, M. Kappl, Force measurements with the atomic force microscope: Technique, interpretation and applications, Surf. Sci. Rep. 59 (2005) 1-152.

[20] E. Chung, H. Kweon, S. Yiacoumi, I. Lee, D.C. Joy, A.V. Palumbo, C. Tsouris, Adhesion of spores of Bacillus thuringiensis on a planar surface, Environ. Sci. Technol. 44 (2010) 290-296.

[21] H. Kweon, S. Yiacoumi, C. Tsouris, Friction and adhesion forces of bacillus thuringiensis spores on planar surfaces in atmospheric systems, Langmuir 27 (2011) 14975-14981. 
[22] H. Kweon, S. Yiacoumi, I. Lee, J. McFarlane, C. Tsouris, Influence of surface potential on the adhesive force of radioactive gold surfaces, Langmuir 29 (2013) 11876-11883.

[23] E. Chung, S. Yiacoumi, I. Lee, C. Tsouris, The role of the electrostatic force in spore adhesion, Environ. Sci. Technol. 44 (2010) 6209-6214.

[24] I. Lee, E. Chung, H. Kweon, S. Yiacoumi, C. Tsouris, Scanning surface potential microscopy of spore adhesion on surfaces, Colloid. Surface. B 92 (2012) 271-276.

[25] L. Boyer, F. Houze, A. Tonck, J.L. Loubet, J.M. Georges, The influence of surface-roughness on the capacitance between a sphere and a plane, J. Phys. D Appl. Phys. 27 (1994) 1504-1508.

[26] N. Elfstrom, R. Juhasz, I. Sychugov, T. Engfeldt, A.E. Karlstrom, J. Linnros, Surface charge sensitivity of silicon nanowires: Size dependence, Nano Lett. 7 (2007) 2608-2612.

[27] I.G. Higginbotham, R.H. Williams, A.J. McEvoy, Metal/non-metal interfaces: Adhesion of gold on mica, Journal of Physics D-Applied Physics 8 (1975) 1033-1043.

[28] R.M. Lyndenbell, The interaction of crystal-surfaces in close proximity, Surface Science 244 (1991) 266-276.

[29] L. Xu, M. Salmeron, Effects of surface ions on the friction and adhesion properties of mica, Langmuir 14 (1998) 2187-2190.

[30] I.G. Higginbotham, R.H. Williams, A.J. McEvoy, Metal/non-metal interfaces: Adhesion of gold on mica, J. Phys. D Appl. Phys. 8 (1975) 1033-1043.

[31] N. Alcantar, J. Israelachvili, J. Boles, Forces and ionic transport between mica surfaces: Implications for pressure solution, Geochim. Cosmochim. Ac. 67 (2003) 1289-1304.

[32] H. Sugimura, Y. Ishida, K. Hayashi, O. Takai, N. Nakagiri, Potential shielding by the surface water layer in Kelvin probe force microscopy, Applied Physics Letters 80 (2002) 1459-1461.

[33] C.A. Rezende, R.F. Gouveia, M.A. da Silva, F. Galembeck, Detection of charge distributions in insulator surfaces, J. Phys.-Condens. Mat. 21 (2009).

[34] J. Israelachvili, Intermolecular and surface forces, Academic Press, London, 1991. 
[35] D. Beaglehole, H.K. Christenson, Vapor adsorption on mica and silicon: Entropy effects, layering, and surface forces, J. Phys. Chem. 96 (1992) 3395-3403.

[36] D.A. Guzonas, M.L. Hair, Polymer displacement and the role of impurities in homopolymer adsorption: A surface force balance study, Langmuir 7 (1991) 2346-2352.

[37] Z.G. Liu, Z. Li, H.L. Zhou, G. Wei, Y.H. Song, L. Wang, Observation of the mica surface by atomic force microscopy, Micron 36 (2005) 525-531. 